2007-04-01

\title{
User Quality of Experience-aware Multimedia Streaming over Wireless Home Area Network
}

\author{
Gabriel-Miro Muntean \\ Dublin City University, munteang@eeng.dcu.ie \\ Nicola Cranley \\ Technological University Dublin, nicola.cranley@tudublin.ie
}

Follow this and additional works at: https://arrow.tudublin.ie/commcon

Part of the Systems and Communications Commons

\section{Recommended Citation}

Muntean, G., Cranley, N.: User Quality of Experience-aware Multimedia Streaming over Wireless Home Area Network.IEEE 65th Vehicular Technology Conference, 2007. VTC2007-Spring, Dublin, Ireland. 22-25 April 2007.

This Conference Paper is brought to you for free and open access by the Communications Network Research Institute at ARROW@TU Dublin. It has been accepted for inclusion in Conference papers by an authorized administrator of ARROW@TU Dublin. For more information, please contact arrow.admin@tudublin.ie, aisling.coyne@tudublin.ie,gerard.connolly@tudublin.ie.

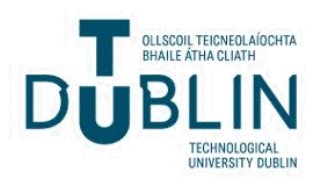




\section{User Quality of Experience-aware Multimedia Streaming over Wireless Home Area Network}

\author{
Gabriel-Miro Muntean \\ School of Electronic Engineering, \\ Dublin City University, \\ Glasnevin, Dublin 9, Ireland \\ munteang@eeng.dcu.ie
}

\begin{abstract}
For multimedia streaming over wireless networks, there is a trade-off between the capacity of the wireless links and the end-user perceived-quality, which can be affected by the compression scheme used, content characteristics and adaptation algorithm (if any). In this paper, this trade-off is investigated for streaming various motion content multimedia over an IEEE 802.11b-based Wireless-Home Area Network using the QualityOriented Adaptation Scheme (QOAS). QOAS performance is compared to that of a non-adaptive scheme when using MPEG-2 and MPEG-4 encoding in terms of average end-user perceived quality, number of streaming sessions concurrently supported, loss rate, delay, jitter and total throughput. Simulation results show that by using QOAS and MPEG-4 encoded streams a much higher number of concurrent streams are supported at an average quality above "good" level on the ITU-T five-point quality scale in comparison with other situations. In this case all the other streaming performance parameters were also significantly better.
\end{abstract}

Keywords-Adaptive video streaming, Wireless Home Area Network, grading scheme, end-user perceived quality.

\section{INTRODUCTION}

Lately broadband connectivity to home residences has enabled access to high-speed Internet as well as to useroriented rich media content services that allow for the distribution of high quality multimedia streams (e.g. digital and interactive TV, Video on Demand, videoconferencing, gaming, etc.) [1]. Currently multimedia streaming is mainly performed via wired IP infrastructures to single computerbased delivery points. At the same time customers are still served by many home appliances interconnected by wires, which provide a single localized service and require user presence in the neighborhood of the end-device.

A recent survey in the U.S. found that many customers have either adopted (around 7 million homes in the U.S. alone) or expressed their intentions of installing wireless technology in their homes (more than 49\% of people surveyed) [2, 3]. This is due to the many advantages of wireless technology over its wired counterpart, such as flexibility of viewer location, mobility and convenience. In this context, wireless solutions support building of an in-home service delivery infrastructure [4] in the form of a WHAN (Wireless Home Area Network). WHANs can be used to interconnect home computers, telephones, home theatres and any other home device and so enable the distribution of rich content, such as multimedia, to users anywhere in the house, anytime and to any device.

\author{
Nikki Cranley \\ School of Electronic \& Communications Engineering, \\ Dublin Institute of Technology, \\ FOCAS Institute, Dublin 8, Ireland \\ Nikki.Cranley@CNRI.DIT.ie
}

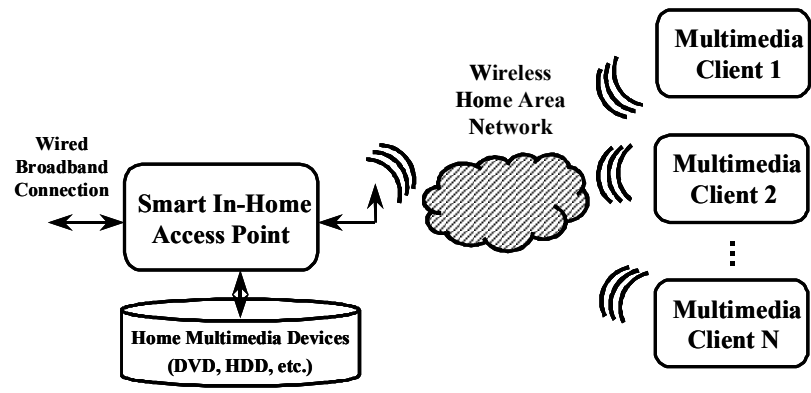

Fig. 1. Wireless in-home multimedia stream distribution architecture

A typical WHAN-based solution for multimedia-based content distribution is presented in Figure 1. The Smart InHome Access Point acts as a local server and provides services on demand to remote Multimedia Clients via WHAN. The multimedia-based content is either acquired via the Wired Broadband Connection or is streamed from a home-located source such as DVD player, hard disk, etc.

However there are many performance related issues when using wireless networks. The main difficulty is that wireless networks allow for much lower delivery rates than wired networks where, typically up to $100 \mathrm{Mbps}$ can be supported. For example a wireless IEEE 802.11b network can support data rates up to $11 \mathrm{Mbps}$, whereas using IEEE 802.11g up to $54 \mathrm{Mbps}$ can be reached. Yet in practice only half of the advertised bit rate can be achieved. Wireless networks are particularly error-prone and since they use radio waves, the data signals are subject to attenuation with distance and signal interference. In addition, the transmission quality is also affected by contention between users who are attempting to access and transmit data on the shared radio channel. This contention results in users having to wait until their backoff process is complete before they can access the channel. All these factors ultimately affect end-user perceived quality or Quality of Experience (QoE).

As QoE is difficult to assess, research has focused on easier-to-measure performance-related Quality of Service (QoS) parameters. Several approaches [5] were proposed in order to provide certain level of QoS when streaming multimedia over wired networks with variable delivery conditions. By using adaptive solutions such as TFRCP [6], LDA+ [7], RAP [8], RLM [9] or RLC [10] good QoS-related results were obtained in highly loaded wired networks. However, none of these schemes addressed the effect on enduser QoE. Quality Oriented Adaptation Scheme (QOAS) was 
proposed in [11] and showed a significant improvement on these schemes for both subjective and objective testing [12].

More recently, diverse solutions were proposed for scalable multimedia transmissions [13] over wireless access networks [14] or wireless ad-hoc networks [15]. Many of these adaptive solutions gradually vary the video streams' characteristics in response to fluctuating network conditions thereby allowing for the perceived quality to be gracefully adapted. Among the proposed solutions are layer-based schemes such as $[14,16]$, object-based adaptation mechanisms [17], fine-granular scalability-based solutions [18] and perception-based approaches [19]. Admission and error control that are used along with the adaptive solutions in either centralized [15] or distributed approaches [20], are employed in order to increase their effectiveness. Complementing these approaches, the QoS capabilities offered by the emerging IEEE 802.11e standard may significantly improve users' QoE by allowing for multiple-priority-based distribution of multimedia content.

This paper presents performance testing results in terms of average user QoE, number of streaming sessions concurrently supported, loss rate, delay, jitter and total throughput when streaming MPEG-2 and MPEG-4-encoded multimedia using QOAS and a non-adaptive approach over an IEEE 802.11bbased WHAN. In the next section, QOAS is briefly described and test results are presented and discussed. Then conclusions are drawn and directions for future work are indicated.

\section{QUALITY ORIENTED ADAPTATION SCHEME (QOAS)}

\section{A. QOAS-Overview}

QOAS is an unicast rate-based adaptive scheme for multimedia streaming that maximises user QoE in existing delivery conditions [11]. It includes client and server-located components that are involved in the bi-directional exchange of video data and control packets through the delivery network. The client monitors the transmission and user QoE-related parameters using the Quality of Delivery Grading Scheme (QoDGS). QoDGS regularly computes the quality of delivery scores, which are sent as feedback to the server. The Server Arbitration Scheme (SAS) analyses these scores and proposes adjustment decisions in order to increase user QoE in existing delivery conditions.

QOAS defines a number of different server states that are assigned to a different stream quality during each streaming session. For example a five-state model was used for the experimental tests presented in this paper. The stream quality versions differ in terms of compression-related parameters (e.g. resolution, frame rate, colour depth) and therefore have different bandwidth requirements. During transmission the server dynamically varies its state according to the client QoDGS feedback. For example, when the client reports a decrease in end-user quality, the server switches to a lower quality state, which reduces the quantity of data sent. In improved conditions, the server gradually increases the quality of the delivered stream. Figure 2 presents a schematic description of QOAS's adaptation principle for the case of pre-recoded multimedia streaming.

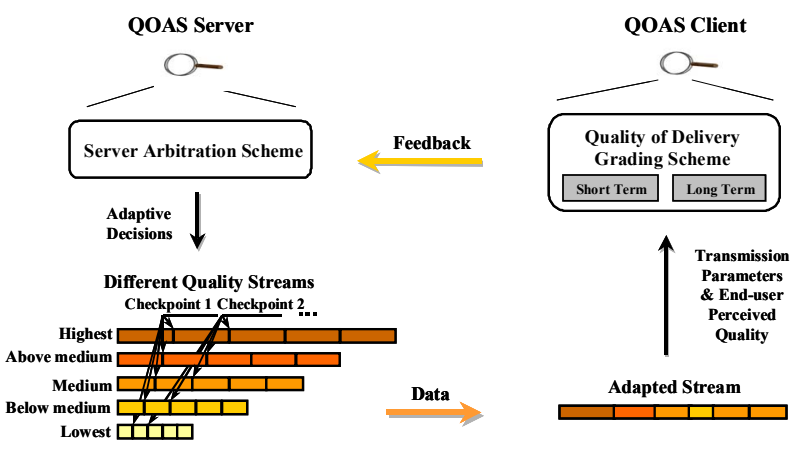

Fig. 2. Schematic description of QOAS's adaptation principle

The client-located QoDGS monitors and evaluates the effect of the delivery conditions on end-user perceived quality. The grading process is based on monitoring both short-term and long-term variations of packet loss rate, delay, and delay jitter, which have been shown to have a significant impact on the received quality. Short-term monitoring is important for learning quickly about transient effects, such as sudden traffic changes, and for quickly reacting to them. The long-term variations are monitored in order to track slow changes in the overall delivery environment, such as new users in the system. QoDGS also takes into account user QoE as measured by the no-reference moving picture quality metric Q [21], which maps the joint impact of bitrate and data loss on video quality onto the ITU-T R P.910 five-point grading scale [22]. More details about QoDGS are presented in [11].

The server-located SAS considers the values of a number of consecutive QoDGS scores from the client and, by averaging these values, asymmetrically suggests adjustment decisions. It requires fewer scores to trigger a quality decrease than for a quality increase, ensuring a fast reaction during bad delivery conditions and helping to eliminate its cause. An increase is performed only when the network conditions have improved. This asymmetry helps also to maintain system stability, by reducing the frequency of quality variations.

\section{B. QOAS - Deployment}

For testing QOAS performance when streaming multimedia over WHAN, the server-side QOAS component is deployed at the Smart In-Home Access Point level, whereas the client-side QOAS component at the Multimedia Client level.

In order to adaptively react fast enough to the highly dynamic variations of the delivery conditions when streaming over wireless networks, there is a need for accurate information from the client at all times. Therefore QOAS employs a very high feedback frequency with small feedback report packets (40 B) that are sent every $100 \mathrm{msec}$. This value balances the need for the most up-to-date information with the requirement of low overhead. Both the QoDGS short-term and long-term monitoring periods and are respectively set to an order and two orders of magnitude greater than the feedbackreporting interval.

Adaptive decisions must also to be taken quickly and therefore SAS upgrade period was set to $6 \mathrm{sec}$ whereas the downgrade timeout used was $1 \mathrm{sec}$. These values ensure both protection against any noise that may occur in the grading scheme and the QoDGS's asymmetry in the grading process. 


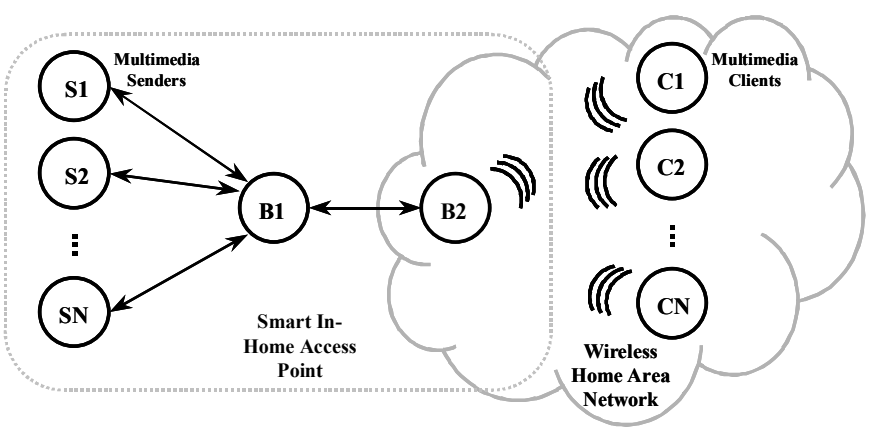

Fig. 3 Simulation Setup

\section{EXPERIMENTAL TESTING RESUlts}

\section{A. Simulation Models, Setup and Video Sequences}

The experimental tests performed consisted of simulations using models for QOAS and Non-Adaptive (NoAd) schemes built using Network Simulator version 2.27 (NS-2) [23]. The topology used for simulations is presented in Figure 3 and reflects the architecture for in-home distribution of multimedia-based content shown in Figure 1. It assumes a single Smart in-Home Access Point (SHAP) that streams multimedia content to a number of $\mathrm{N}$ Multimedia Clients (deployed at nodes $\mathrm{Ci}, \mathrm{i}=1, \mathrm{~N}$ ) over an IEEE 802.11b-based WHAN. SHAP retrieves multimedia data from a number of Multimedia Senders localized at nodes $\mathrm{Si}, \mathrm{i}=1, \mathrm{~N}$. Si-B1 (bandwidth $=100 \mathrm{Mbps}$, propagation delay $=5 \mathrm{msec}$ ) and B1B2 (bandwidth $=200 \mathrm{Mbps}$, propagation delay $=5 \mathrm{msec}$ ) links are over-provisioned so that the only packet drops and significant delays are caused by the delivery over the WHAN. The buffering at the B1-B2 link uses a drop-tail queue of size proportional to the product of round trip time and link bandwidth. Client buffer size was set such as no loss occurs due to buffer length limitation.

All scenarios were implemented and tested in NS-2 using the NOAH (No Ad-Hoc) wireless routing agent for a duration of 500 seconds with a medium bandwidth of $11 \mathrm{Mbps}$. NOAH only supports direct communication between base stations and mobile nodes. The MAC settings used in the simulations are shown in Table I.

Five five-minute long video sequences were selected from movies with different degrees of motion content: $D H$ - high, $J P$ - average, $D W$ - average/low, $F M$ - low and $R E$ - average /high. The clips were encoded at five different rates using an MPEG-2 encoder and MPEG-4 Advanced Simple Profile encoder respectively. In both MPEG-2 and MPEG-4 cases, the frame rate was 25 frames/sec and the IBBP frame pattern was 9 frames/GOP. The MPEG-2 test sequences were encoded at five different bit rate between $2 \mathrm{Mbps}$ and $4 \mathrm{Mbps}$ whilst the MPEG-4 test sequences were encoded at average bitrates between $64 \mathrm{Kbps}$ and $512 \mathrm{Kbps}$. Details about the peak/mean bitrate ratios of all encoded multimedia sequences are presented in Table II.

Multimedia streams were delivered using NS-2-built NoAd and QOAS models. The NoAd model streams multimedia content at maximum encoding rate regardless of the delivery conditions (4 Mbps for MPEG-2 and $512 \mathrm{Kbps}$ for MPEG-4). NoAd does not use any feedback and does not adapt the transmission rate or the transmitted video quality in any way.
TABLE I

MAC SeTtings Used DURING Simulations

\begin{tabular}{|l|l|}
\hline \multicolumn{2}{|c|}{ MAC Settings } \\
\hline Bit Rate & $11 \mathrm{M}$ \\
\hline CW Min & 21 \\
\hline CW Max & 1023 \\
\hline Slot Time & $20 \mathrm{us}$ \\
\hline SIFS & $10 \mathrm{us}$ \\
\hline Preamble Length & 144 \\
\hline Short Retry Limit & 7 \\
\hline Long Retry Limit & 4 \\
\hline \hline
\end{tabular}

TABLE II

Peak/Mean Bitrate Ratios FOR All MPEG-2 AND MPEG-4 ENCODED QUALITY VERSIONS OF THE CLIPS USED DURING SIMULATIONS

\begin{tabular}{c|ccccc|ccccc|}
\hline \hline & \multicolumn{3}{|c|}{ MPEG-2 - Average Rate (Mbps) } & \multicolumn{5}{c|}{ MPEG-4 - Average Rate (Kbps) } \\
\hline Clip & $\mathbf{2 . 0}$ & $\mathbf{2 . 5}$ & $\mathbf{3 . 0}$ & $\mathbf{3 . 5}$ & $\mathbf{4 . 0}$ & $\mathbf{6 4}$ & $\mathbf{1 2 8}$ & $\mathbf{2 5 6}$ & $\mathbf{3 8 4}$ & $\mathbf{5 1 2}$ \\
\hline DH & 7.48 & 7.43 & 6.31 & 5.65 & 4.06 & 3.92 & 3.85 & 4.46 & 4.56 & 4.46 \\
RE & 6.91 & 6.51 & 6.23 & 6.12 & 6.05 & 6.86 & 4.50 & 4.32 & 4.31 & 4.31 \\
DW & 5.56 & 4.51 & 4.36 & 4.08 & 3.56 & 4.18 & 3.91 & 3.90 & 3.90 & 3.90 \\
JP & 4.83 & 4.38 & 4.04 & 3.71 & 3.41 & 4.63 & 3.26 & 3.20 & 3.19 & 3.19 \\
FM & 3.99 & 3.67 & 3.42 & 3.09 & 2.93 & 4.75 & 3.79 & 3.78 & 3.78 & 3.78 \\
\hline \hline
\end{tabular}

The QOAS model conforms to the system described in Section II, using $100 \mathrm{msec}$ inter-feedback intervals and a five state server adaptation model.

\section{B. Simulation Scenarios and Results}

Simulations were deployed using the topology described in Section III.A where a number of clients randomly select both the movie clip and the starting sequence from within the chosen clip. This ensures that all movie types are used during streaming process and the independence of the simulation result from the natural bit rate variation in time within each of the streamed movies. The tests were repeated with an increasing number of clients.

The resulting video streaming processes began in quick succession and ended similarly, which is typical of a number of people availing from multimedia-based services starting at some fixed time. The transitory periods of $5 \mathrm{sec}$ duration are not considered in this paper. During the stable periods loss rate, delay, jitter, bitrate and user QoE were measured and average values were computed and analysed.

The QOAS and NoAd approaches were used in turn as the video streaming method and content encoded using MPEG-2 and MPEG-4 encoding schemes respectively was delivered to a number of $\mathrm{N}$ multimedia clients. In successive tests $\mathrm{N}$ was gradually increased from 1 to 10 . The tests were not performed with more than 10 simultaneous multimediastreaming sessions as at this level the average user QoE is just above the "good" perceptual level on the ITU-T R. P.910 fivepoint subjective quality scale, which was considered here as the minimum level of interest.

Figure 4 presents a comparison between the average estimated end-user QoE as a function of the increase in the number of simultaneously served clients in all simulated situations. The end-user perceived quality was measured by the no-reference moving pictures quality metric Q [22] on the ITU-T R P.910 five-point grading scale [23]. Figure 5 shows how the average loss rate varies as a function of the increase in the number of simultaneous streamed multimedia clips. 


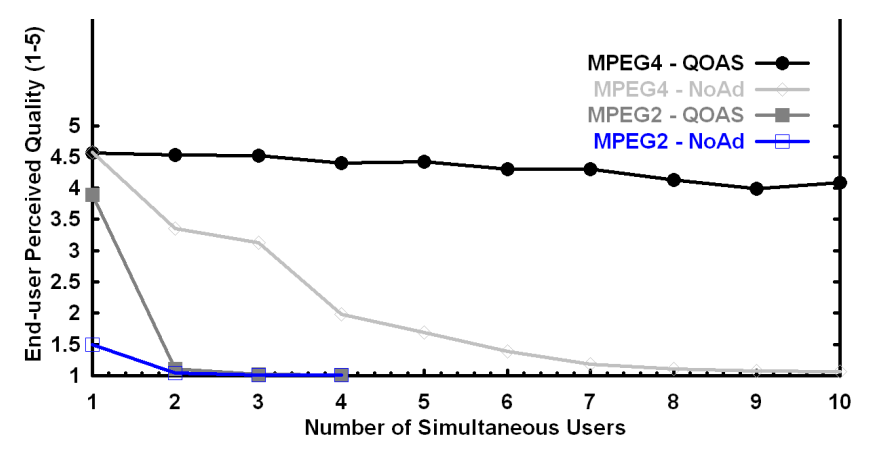

Fig. 4. Quality

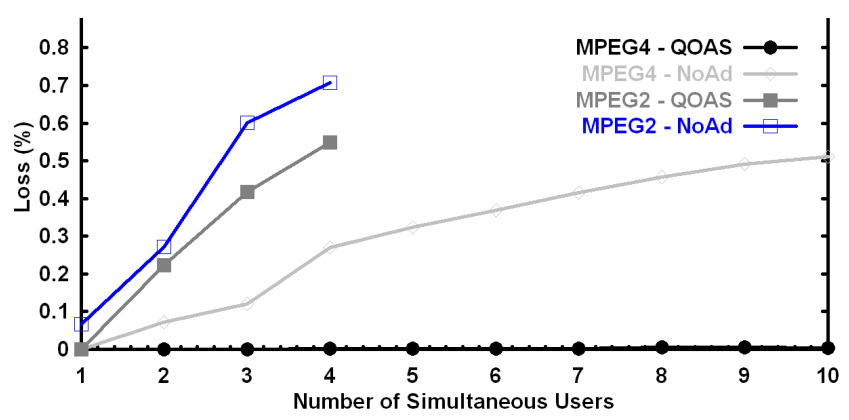

Fig. 5. Loss

Figure 6 and Figure 7 plot the average delay and jitter when the number of concurrent multimedia streaming sessions gradually increases from 1 to 4 when using MPEG-2 encoded multimedia content and from 1 to 10 when using MPEG-4.

These test results show that the number of simultaneous multimedia-based stream deliveries is significantly higher when using QOAS in comparison with the NoAd approach given certain targeted end-user quality level. For example, to maintain a "good" perceptual quality level, by using QOAS five times more client devices could be served with MPEG-4encoded multimedia-based content than by using NoAd approach. However it is clear that in spite of the QOAS's adaptiveness, if the target is set at "good" quality level, only one MPEG-2-encoded stream can be delivered over the IEEE 802.11b-based WHAN and not even a single NoAd stream.

By analysing the results plotted in Figure 5 that presents the loss rate evolution with the increase in the number of concurrent streaming sessions, one can see how QOAS clearly outperforms NoAd solution when streaming MPEG-4 encoded content, successfully maintaining a loss rate very close to the ideal $0 \%$ in comparison with NoAd's loss rate of over $0.6 \%$.

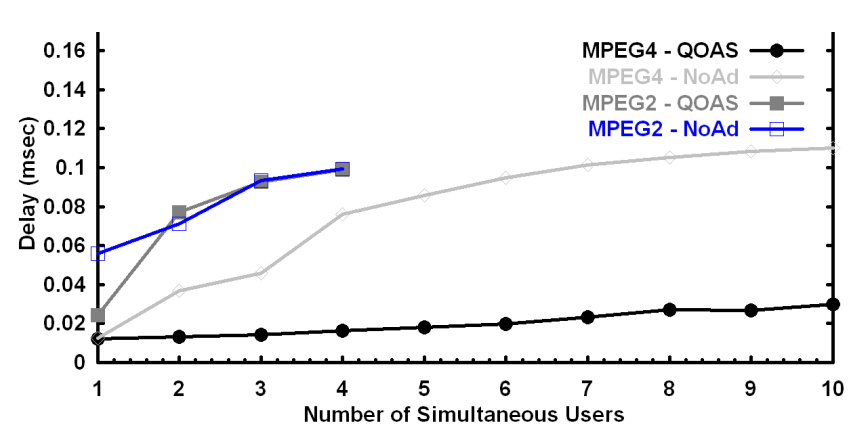

Fig. 6. Delay

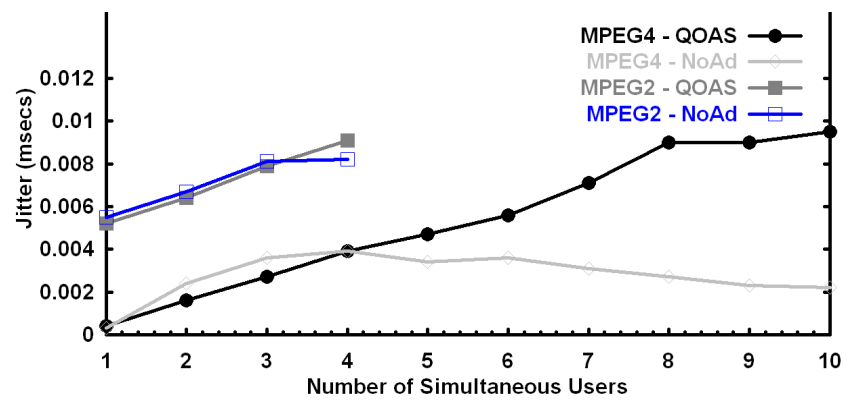

Fig. 7. Jitter

The plot also indicates that despite the lower loss rates achieved using QOAS in comparison with NoAd, MPEG-2encoded streams cannot be transmitted over IEEE 802.11bbased WHAN and expect high user QoE. Therefore higher bandwidth wireless solutions such as IEEE $802.11 \mathrm{~g}$ for instance are required.

By analysing the delay and jitter variations with increasing numbers of concurrent multimedia streaming sessions over WHAN (Figure 6 and Figure 7), the expected increase in both performance parameters' average values when streaming MPEG-2-encoded content can be verified. However when streaming MPEG-4 content, it is highly significant when analysing the QOAS performance, that the delay remains at very low levels in spite of the high increase in the number of simultaneous multimedia deliveries. In contrast, streaming using the NoAd approach incurs a six fold increase in the delay. The increase in jitter when streaming MPEG-4 content using QOAS with the high increase in traffic over WHAN is normal, as is also the decrease in jitter for the NoAd streaming that corresponds to the high increase in loss. More detailed results are presented in Table III that show the variations in

TABLE III

COMPARISON BETWEEN QOAS AND NOAD PERFORMANCE-RELATED RESULTS

\begin{tabular}{c|ccccc|ccccc|}
\hline \hline & \multicolumn{4}{|c|}{ QOAS (MPEG-4) } & \multicolumn{4}{c|}{ NoAd (MPEG-4) } \\
\hline Clients & $\begin{array}{c}\text { Quality } \\
(\mathbf{1 - 5})\end{array}$ & $\begin{array}{c}\text { Loss Rate } \\
(\mathbf{\%})\end{array}$ & $\begin{array}{c}\text { Delay } \\
(\mathbf{m s})\end{array}$ & $\begin{array}{c}\text { Jitter } \\
(\mathbf{m s})\end{array}$ & $\begin{array}{c}\text { Throughput } \\
(\mathbf{M b p s})\end{array}$ & $\begin{array}{c}\text { Quality } \\
(\mathbf{1 - 5})\end{array}$ & $\begin{array}{c}\text { Loss Rate } \\
(\mathbf{\%})\end{array}$ & $\begin{array}{c}\text { Delay } \\
(\mathbf{m s})\end{array}$ & $\begin{array}{c}\text { Jitter } \\
(\mathbf{m s})\end{array}$ & $\begin{array}{c}\text { Throughput } \\
(\mathbf{M b p s})\end{array}$ \\
\hline $\mathbf{1}$ & 4.57 & 0.00 & 12 & 0.42 & 0.46 & 4.57 & 0.00 & 12 & 0.33 & 0.46 \\
$\mathbf{2}$ & 4.53 & 0.02 & 13 & 1.57 & 0.90 & 3.36 & 7.13 & 37 & 2.40 & 0.60 \\
$\mathbf{3}$ & 4.52 & 0.04 & 14 & 2.67 & 1.35 & 3.13 & 12.01 & 46 & 3.59 & 0.82 \\
$\mathbf{4}$ & 4.40 & 0.12 & 16 & 3.85 & 1.74 & 1.98 & 26.99 & 76 & 3.91 & 0.51 \\
$\mathbf{5}$ & 4.43 & 0.08 & 18 & 4.68 & 2.19 & 1.69 & 32.38 & 86 & 3.41 & 0.54 \\
$\mathbf{6}$ & 4.30 & 0.21 & 20 & 5.63 & 2.54 & 1.39 & 36.79 & 95 & 3.56 & 0.53 \\
$\mathbf{7}$ & 4.30 & 0.17 & 23 & 7.14 & 2.96 & 1.19 & 41.50 & 101 & 3.06 & 0.53 \\
$\mathbf{8}$ & 4.13 & 0.44 & 27 & 8.97 & 3.21 & 1.10 & 45.65 & 105 & 2.66 & 0.56 \\
$\mathbf{9}$ & 3.99 & 0.59 & 27 & 9.02 & 3.45 & 1.07 & 49.04 & 108 & 2.25 & 0.62 \\
$\mathbf{1 0}$ & 4.09 & 0.38 & 30 & 9.50 & 3.95 & 1.06 & 51.02 & 110 & 2.19 \\
\hline \hline
\end{tabular}


average perceived quality, loss (expressed as percentage), delay (msec), jitter (msec) and total throughput (Mbps) with increased numbers of simultaneous connections.

A very significant result was obtained in terms of total throughput that increased six times when using QOAS for delivering MPEG-4 encoded content in comparison with when NoAd was used. This confirms that the adaptation to the increased delivery conditions determined a significant increase in the WHAN overall delivery efficiency for the benefit not only of the end-users, but also of the network operators and service providers. They could increase their revenues by serving using QOAS a higher number of customers that experience the same "good" perceived quality from the existing infrastructure.

\section{CONCLUSIONS AND FURTHER WORK}

This paper presents a comparison of significant performance parameters when streaming MPEG-2 and MPEG-4 encoded multimedia content using the Quality Oriented Adaptation Scheme (QOAS) and a Non-Adaptive (NoAd) scheme respectively over an IEEE 802.11 b-based Wireless Home Area Network (WHAN). The comparison is performed in terms of average end-user perceived quality, number of streaming sessions concurrently supported, loss rate, delay, jitter and total throughput.

Simulation results show that for the same average end-user quality, QOAS can accommodate a significantly higher number of simultaneous streaming sessions while also having higher total throughput. For the same number of concurrent streaming sessions, the estimated average end-user perceived quality is always higher for QOAS than for NoAd. Tests also show that IEEE $802.11 \mathrm{~b}$-based WHAN cannot support more than one concurrent streaming session if MPEG-2-encoded clips are used and where high end-user QoE is required even if a QOAS-based adaptive approach is used for the delivery of multimedia content. However if MPEG-4 encoding scheme is used, QOAS enables the delivery of up to 10 simultaneous streaming sessions over WHAN and the end-user QoE is maintained above the "good" level on the ITU-T R. P.910 five-point perceptual scale. This represents a five-fold increase than when NoAd is used. Furthermore, a six-fold increase in total throughput over the same WHAN in comparison with NoAd is obtained when using QOAS for multimedia streaming. This increase in operational efficiency allows service providers and network operators to maximise their revenues by offering multimedia-based services to an increased number of clients while maintaining a minimum "good" target quality level. Other streaming performance parameters such as loss, delay and jitter also record better average values when using QOAS for streaming MPEG-4encoded streams.

Currently work is in progress to investigate the performance of the QOAS system when streaming multimedia content in the presence of other traffic in order to understand the effects different traffic types have on each other in a WHAN environment. In addition, we plan to determine and optimize the configurable QoS parameters of IEEE 802.11e so as to maximize performance of multimedia delivery whilst behaving fairly with other traffic. Further work will include comparisons of QOAS with other adaptive schemes in similar delivery conditions. It is also planned to carry out subjective perceptual tests on a prototype system to verify the end-user quality results gathered from simulations.

\section{REFERENCES}

[1] S. Dravida, D. Gupta, S. Nanda, K. Rege, J. Strombosky, M. Tandon, "Broadband Access over Cable for Next-Generation Services: A Distributed Switch Architecture", IEEE Communications Magazine, vol. 40, no. 8, August 2002, pp. 116-124.

[2] Consumer Electronics Association, Digital America Home Networking, [Online] Available: http://www.ce.org/publications/books_references/ digital_america/home_networking/wireless_networking.asp

[3] D. Becker, "Wi-Fi overtakes Ethernet for home networks", CNET News.com, $21^{\text {st }}$ Jan. 2005, [Online] Available: http://news.zdnet.co.uk/ communications/wireless/0,39020348,39185187,00.htm

[4] C. Ngo, "A Service-oriented Wireless Home Network", IEEE Consumer Communications and Networking Conference, Jan. 2004, pp. 618 - 620.

[5] D. Wu, Y. T. Hou, W. Zhu, Y.-Q. Zhang, J. M. Peha, "Streaming Video over the Internet: Approaches and Directions", IEEE Trans. On Circuits and Systems for Video Technology, vol. 11, no. 3, 2001, pp. 282-300

[6] J. Padhye, J. Kurose, D. Towsley, R. Koodli, "A Model Based TCP Friendly Rate Control Protocol", Proc. NOSSDAV, 1999.

[7] D. Sisalem, A. Wolisz, "LDA+ TCP-Friendly Adaptation: A Measurement and Comparison Study", Proc. NOSSDAV, 2000.

[8] R. Rejaie, M. Handley, D. Estrin, "RAP: An End-to-end Rate-based Congestion Control Mechanism for Realtime Streams in the Internet", Proc. of INFOCOM, March 1999

[9] S. McCanne, Van Jacobson, M. Vetterli, "Receiver-Driven Layered Multicast", Proc. of SIGCOMM, USA, Aug. 1996, pp. 117-130

[10] L. Vicisano, J. Crowcroft, L. Rizzo, "TCP-like Congestion Control for Layered Multicast Data Transfer", Proc. INFOCOM, vol. 3, March 1998

[11] G.-M. Muntean, P. Perry, L. Murphy, "A New Adaptive Multimedia Streaming System for All-IP Multi-Service Networks", IEEE Trans. on Broadcasting, vol. 50, no. 1, March 2004, pp. 1-10

[12] G.-M. Muntean, P. Perry, L. Murphy, "Subjective Assessment of the Quality-Oriented Adaptive Scheme", IEEE Trans. on Broadcasting, vol. 51, no. 3, September 2005 , pp. 276-286

[13] D. Wu, T. Hou, Y.-Q. Zhang, "Scalable Video Coding and Transport over Broadband Wireless Networks", Proc. of the IEEE, vol. 89, no. 1, Jan. 2001, pp. 6-20

[14] L. Qiong, M. van der Schaar, "Providing Adaptive QoS to Layered Video over Wireless Local Area Networks Through Real-time Retry Limit Adaptation", IEEE Trans. on Multimedia, vol. 6, no. 2, April 2004, pp. 278-290

[15] S. H. Shah, K. Chen, K. Nahrstedt, "Dynamic Bandwidth Management for Single-hop Ad Hoc Wireless Networks", Proc. IEEE Int'l. Conf. Pervasive Comp. and Commun., Dallas-Fort Worth, USA, Mar. 2003

[16] R. Rejaie, M. Handley, D. Estrin, "Layered Quality Adaptation for Internet Video Streaming", IEEE Journal on Selected Areas of Communications (JSAC), vol. 18, no. 12, Dec. 2000, pp. 2530 - 2543

[17] K.-A. Cha, "Content Complexity Adaptation for MPEG-4 Audio-Visual Scene", IEEE Trans. Cons. Elec., vol. 50, no. 2, May 2004, pp. 760-765

[18] M. van der Schaar, H. Radha, "Adaptive Motion-compensation Finegranular-scalability (AMC-FGS) for Wireless Video”, IEEE Trans. Circuits Syst. Video Technol, vol. 12 , no. 6, June 2002, pp. 360-371

[19] N. Cranley, L. Murphy, P. Perry, "Optimum Adaptation Trajectories for Streamed Multimedia”, ACM Multimedia Systems Journal, SpringerVerlag, Vol. 10, No. 5, 2005, pp. 392-401

[20] W. Pattara-Atikom, P. Krishnamurthy, S. Banerjee, "Distributed Mechanisms for Quality of Service in Wireless LANs", IEEE Wireless Communications, June 2003, pp. 26-34

[21] O. Verscheure, P. Frossard, M. Hamdi, "User-Oriented QoS Analysis in MPEG-2 Video Delivery”, J Real-Time Imaging, vol. 5, no. 5, Oct. 1999

[22] ITU-T Recommendation P.910, "Subjective Video Quality Assessment Methods for Multimedia Applications”, Sept. 1999.

[23] Network Simulator-2, [Online]. Available: http://www.isi.edu/nsnam/ns/ 